\title{
Ionization Structure of Heavy Metals for Planetary Nebulae
}

\author{
Belay Sitotaw Goshu \\ Department of Physics, Dire Dawa University, Dire Dawa, Ethiopia
}

\section{Email address:}

belaysitotaw@gmail.com

\section{To cite this article:}

Belay Sitotaw Goshu. Ionization Structure of Heavy Metals for Planetary Nebulae. American Journal of Astronomy and Astrophysics. Vol. 6, No. 2, 2018, pp. 39-48. doi: 10.11648/j.ajaa.20180602.12

Received: April 21, 2018; Accepted: May 25, 2018; Published: July 3, 2018

\begin{abstract}
The purpose of this work to reproduces the flux of lines emitted by the ionized gas, resulting in a good agreement between observed and predicted line fluxes, temperatures and electron density with the chemical abundances of ionic species with carbon and oxygen rich planetary. The model has been calculated using clouds of dust grains in order to calculate the electron temperature, density, and emission fluxes. The dust grain with polycyclic aromatic hydrocarbons (PAH) physics combined with thick shell geometry solves the problem of the heating and allows the fitting of fluxes lines, the electron temperatures and the ionization structure of the nebula. Among other lines, we determined line ratio of CII $\lambda 4267 \mathrm{~A}$, [NII] $\lambda 5755 \mathrm{~A}, 6584 \mathrm{~A}, 6548 \mathrm{~A},[\mathrm{SII}] \lambda 4078 \mathrm{~A}, 4070 \mathrm{~A}, 6716 \mathrm{~A}$, and $6731 \mathrm{~A}$ and compared the results with the observation data by changing the abundances of $\mathrm{C}, \mathrm{N}, \mathrm{O}$ and $\mathrm{S}$, which are enhanced by a factor of 2 . The analysis of the ionization structure of the gas, as well as this calculation of the flux of ions, is presented in this work. Therefore, our result revealed that flux lines like [NII] $\lambda 6584 \mathrm{~A}$, [OII] $\lambda 3727 \mathrm{~A}+29 \mathrm{~A}$ and [OIII] $\lambda 5007 \mathrm{~A}$ greater than the observed fluxes. The nebulae are found to have low electron temperature than found by the previous author, but the electron density is high.
\end{abstract}

Keywords: Planetary Nebulae, Abundances, Photoionization, Star Evolution

\section{Introduction}

Planetary nebulae $(\mathrm{PNe})$ are the results of the evolution of low to intermediate mass stars $(0.8-8 \mathrm{M} \odot)$ [1]. These stars experience a phase of extreme mass loss during the previous asymptotic giant branch (AGB) that causes the ejection of the stellar envelope [2]. When this mass loss ceases the AGB phase ends and the star evolves into a short-lived evolutionary stage called the 'post AGB' or 'proto-PN' phase just before the star becomes a Planetary Nebulae (PN) [1]. The formation of axisymmetric structures in PNe (ranging from elliptical to bipolar) is believed to be completed by the end of the AGB phase $[1,3,4]$.

There is different asymmetric morphology from elliptical too bipolar to study the PNe but it is unknown. The first is the interaction of stellar winds, binary systems as central stars $[3,5]$, non-radial pulsation $[1,6]$ or the influence of magnetic fields as it was described in $[7,8]$. To identify which one (s) of the above is the dominant process, in this morphology as early as possible after the departure from the spherical symmetry takes place in PNe.

The knowledge of chemical abundances in Planetary
Nebulae (PNe) is important since they can be used as constraints in order to study the emission line fluxes, as well as for studies of the ionization structure of planetary nebulae. Since this work is approached by means of photoionization models, trying to reproduce the measured fluxes and ionization structure of nebulae of the lines emitted by the ionized gas under different assumptions about its metal content and ionizing incident radiation.

The purpose of this work is to greatly expand the work of [9] on planetary nebulae models by creating functionality allowing the existing spectral simulation with the code cloudy to produce predicted spectra. Cloudy used to produce the existing emission lines and ionization structure of nebulae with different basic assumptions and parameters. Only the ionized part of the nebula is considered for modeling, which implies that matching the observed nebular line fluxes is given a more serious consideration than matching the infrared radiation by dust though dust grains have been introduced in the ionized nebula of the model. Moreover, in this paper we analyze the effect of geometry on the electron density and electron temperature with the abundances of chemicals. In fact, the previous paper was done by [9], and analyzes the effect of chemical on the electron temperature. 
The organization of this paper is as follows. The first section describe the properties of the planetary nebulae database from Infrared Astronomical Satellite (IRAS) 070277934 as given in the literature, focusing on the main characteristics and gas physical conditions. Section 3 describes the photoionization model has used with the inclusion of the input stellar ionizing population and the effects of dust on emission fluxes. Section 4 presents discussion of the results in comparisons with the observations. Finally, the conclusions of this work are presented in section 5 .

\section{IRAS 07027-7934}

IRAS 07027 is a very peculiar young PN. It has a central star that was classified by $[1,10]$. As of Wolf-Rayet Carbon type [WC], there about half a dozen $\mathrm{PNe}$ that has been classified as [WC11] [11, 12]. They all have stellar temperatures between 28000 and $35000 \mathrm{~K}$ and are supposed to be in the earliest observable PN evolution. It is one of among the brightest IRAS PNe and it has IRAS colors similar to other young PNe. The youth of IRAS 07027 as a $\mathrm{PN}$ is also evidenced by the detection of $\mathrm{OH}$ maser emission at $1612 \mathrm{MHz}$, which is usually observed in their precursors, the $\mathrm{OH} / \mathrm{IR}$ stars, but rarely in $\mathrm{PNe}$. The $\mathrm{OH}$ emission is being detected only coming from the blue side of the shell, as consequence of the ionized inner region being optically thick at $1612 \mathrm{MHz}[13,14]$. This is supported by the shifting velocity with respect to the $\mathrm{CO}$ emission, which is detected by expansion velocity which was moving at by $30.5 \mathrm{~km} / \mathrm{sec}$, which twice of expansion velocity given by $[1,15]$. This is because the ionized gas is located inside molecular cavity; $\mathrm{CO}$ can expand by moving with velocity in the range between $15 \mathrm{~km} / \mathrm{se}$ to $70 \mathrm{~km} / \mathrm{s}$ along the polar directions of axis [16].

PNe can be quite strong mid-IR emitting objects because of PAH (polycyclic aromatic hydrocarbon) emission, fine structure lines, high excitation mid-IR lines like [OIV] 25.89 $\mu \mathrm{m}, \mathrm{H}_{2}$ molecular lines and thermal dust emission within the nebulae and in circumnuclear disks. Such emissions make them a decent prospect for uncovering them as mid-IR sources [17]. IRAS 07027 has dust PAH, which detects the strong emission of the spectrum. It includes oxygen with carbon-rich clouds of dust. IRAS 07027 is the only WC stars, which belong to the rare $\mathrm{PNe}$ with $\mathrm{OH}$ maser emission that links $\mathrm{OH} / \mathrm{IR}$ stars with carbon-rich PNe [1]. Although the central star is carbon rich $(\mathrm{C} / \mathrm{O}>1)$, the hydrogen deficient is found to be oxygen rich, a situation not predicted by the single star 'born-again' theory of its formation [18].

\section{Model Description}

The model here described simulates the properties of the ionized gas for planetary nebulae with IRAS 07027-7934.
More than $90 \%$ of the entire sky was surveyed by IRAS, and large numbers of late-type stars were with stellar winds were observed $[19,20]$. PNe has been calculated using photoionization code cloudy (version c13.03) [21]. This model is characterized mainly by a set of input parameters including the gas density, chemical abundances, and wind velocity, which differ from [9] by the effective temperature, radius, and chemical abundances. The main properties of the fitting model are summarized in Table 1. The photoionization models do not yet include all the physical processes needed to reproduce all the ratios observed in real nebulae. The previous model did not include the possible presence of stellar winds speed due to WR stars in [9].

\subsection{Input Models}

The essence of the chemical evolution of this planetary can be illustrated by imagining a certain space containing nebulae gas of primordial composition situated at any arbitrary location within a galaxy. As portions of the gas collapse, fusion processes within the stars that are formed convert hydrogen into heavier elements, and this chemically enriched material is subsequently expelled into the interstellar medium through stellar winds of $30 \mathrm{~km} / \mathrm{s}$, planetary nebula formation. This was done by photoionization model of planetary nebula [12]. As a result, there are mixed of gas with the composition of matter found near the galaxies so that the abundances of helium and other heavy elements increase relative to hydrogen [23].

The existence of planetary nebulae requires the presence of a circumstellar envelope of relatively high density $\left(>10^{2}\right.$ $\mathrm{cm}^{3}{ }^{3}$ ) [24] density as well as a central star of temperature greater than $30000 \mathrm{~K}$ [20].

While this problem seems to be intractably complicated, certain approximations are possible. First, the ionized component is heated by the central star alone and is not affected by the neutral components. Second, since most of the dust radiation is emitted in the far-infrared where the nebula is optically thin, there is very little feedback of the dust emission into the other components. PAHs make up the low-mass end of the grain size distribution [25]. The bulk infrared emission features of PAHs have been detected in interstellar spectra, but it is still impossible to identify individual molecules [24, 5]. Similarly, the molecular gas emits only line radiation; although these lines can be optically thick because of self-absorption, they do not constitute a significant source of heating of the dust component. However, near-infrared emission from the dust can be absorbed by the molecular gas via vibrations excitation, and the molecular line transfer problem is dependent on the output of the dust transfer problem. The model parameters, as well as the physical properties for the models, are summarized in Table 1 , and discussed in more detail below. 
Table 1. Parameters of planetary nebulae model.

\begin{tabular}{ll}
\hline Parameters & Values \\
\hline$T_{\text {eff }}$ & $30,500 \mathrm{~K}$ \\
Radius of the Star & $\log \left(R_{\odot}\right)=6.5 R_{\odot}=11.66 \mathrm{~cm}$ \\
Density & $\log N(H)=4.74 \mathrm{~cm}^{-3}$ \\
Abundances & $\mathrm{He}=11.0, \mathrm{C}=8.48, \mathrm{~N}=7.85, \mathrm{O}=8.61, \mathrm{Ne}=7.78, \mathrm{~S}=7.11$ and Ar $=6.46$ \\
Filling factor & $0.5, \alpha=-0.5$ \\
Size distribution & Single \\
Dust & grains Orion, grains Orion \\
Grains & PAH, function 3 \\
Inner radius & $\log r_{i}=17.5 \mathrm{~cm}$ \\
Wind speed & $30 \mathrm{~km} / \mathrm{sec}$ \\
\hline
\end{tabular}

This paper mainly focuses on planetary nebulae since these best tracks used to study the chemical evolution of the interstellar medium. It has used the chemical abundances' which were discussed in [26-28] and these agree with the values given in Table 1. Initially the abundances and densities were adopted from Table 1. Initially, the abundances of chemical elements were adopted from [29]. The abundances elements by number relative to hydrogen are $\mathrm{He} / \mathrm{H}=-1.00, \mathrm{C} / \mathrm{H}=3.02(-4), \mathrm{N} / \mathrm{H}=7.08(-5), \mathrm{O} / \mathrm{H}=4.07$ $(-4), \mathrm{Ne} / \mathrm{H}=6.03(-5), \mathrm{S} / \mathrm{H}=1.29(-5)$ and $\mathrm{Ar} / \mathrm{H}=2.88(-6)$, although all of the lightest 30 elements are not included in this calculation. The assumed composition has little effect on the emission line fluxes and ionization structure of elements. The abundances of $\mathrm{O}, \mathrm{Ne}, \mathrm{S}$ and $\mathrm{Ar}$ are most readily (but not exclusively) observed in emission-line objects such as planetary nebulae shown [30]. As it has computed a series of models in which the abundances were decreased to the very low metallicities appropriate for low-mass galaxies. The main effect of this composition is on the emission-line spectrum, which is used to change the equilibrium electron temperature [31]. The effective temperature $T_{e f}=30,500 \mathrm{~K}$ and radius of the star is $\log \left(R_{\odot}\right)=6.5 R_{\odot}=11.66 \mathrm{~cm}$.

By default, Cloudy stops when the model reaches an electron temperature of $4000 \mathrm{~K}$ [21]. However, some of the models with high metallicity reach this temperature in the fully ionized zone; we switched off this criterion and used the lowest temperature of the electron to be $100 \mathrm{~K}$ as stopping criteria [21].

\subsection{The Basic Assumptions}

A number of simplifying assumptions are taken this model. It essentially to assume a spherically symmetric and static nebula in steady state and allows the presence of dust grains mixed with the gas. The diffuse radiation field is treated under the on-the-spot (OTS) approximation. To take care of fluctuations of electron density, the code allows a filling factor of a value less than unity and varies with the power law shown in Eq1. The approach to dust modeling is on the lines of $[25,32,33]$. The grains are assumed to be heated by stellar and $L_{y \alpha}$ line photons and assumed to emit like a blackbody under thermal equilibrium. Effects of scattering are not included and only grains of a single size are treated. The work has also considered the abundances of helium relative to hydrogen is the uniform, there is no evidence to vary the abundances, instead, hydrogen changed into helium by nuclear interactions throughout the process.
In this work it has added a certain amount of dust in order to fit correctly the measured electron temperature. The presence of grains within the ionized gas have some consequence on the physical conditions of the nebulae that should not be neglected, including the depletion of metals in gaseous form on to grains or absorption of energetic radiation. The heating of dust can affect the electron temperature inside the nebulae [8]. Grains will also participate directly in the thermal balance by contributing photoelectrons that heat the plasma and by cooling through radiation of energy transferred in captures of electrons [32, 34]. In order to simplify this, it has assumed default Orion grains and grains $\mathrm{PAH}$ function to study planetary nebulae ionization structure, electron temperature, electron number density and temperatures of grains.

\section{Results and Discussions}

This model is developed based on the parameters shown in Table 1 . The results are shown in Table 2 like CII $\lambda 4267 \mathrm{~A}$, HeI $\lambda \lambda 3889,4471 \mathrm{~A}$, the predicted fluxes and the result we obtained too weak. Moreover, fluxes like [O I] $\lambda \lambda 6300$, 6363Aand [S II] $\lambda 6731 \mathrm{~A}$ were also too weak relative to observed fluxes. Fluxes like [N II] $\lambda \lambda 6584,6548$ A, [O II] $\lambda \lambda 3727+29 \mathrm{~A}$ and [O III] $\lambda 5007 \mathrm{~A}$ are overestimated relative to observed fluxes. The rest fluxes relatively match with observed values. This is due to our basic assumptions we design to model the planetary nebulae.

It is possible to generate different models of each PN satisfying the very strong and numerous observational constraints, as an example of line fluxes CII $\lambda 4267 \mathrm{~A}$, [O I] $\lambda \lambda 6300,6363 \mathrm{~A}$ and [S II] $\lambda 6731 \mathrm{~A}$. Given a $\mathrm{T}_{\text {eff }}$ for the central star, the model parameters are constrained by line fluxes [23]. As we have shown in Table 1, the effective temperature is greater than the temperature given by [9], not only effective temperature but also the chemical abundances and the filling factor. The effective temperature of the star will continue to increase due to the loss of envelope mass as the result of hydrogen shell burning. The Proto Planetary Nebulae (PPN) phase continues until the central star is hot (Te 30 $000 \mathrm{~K}$ ) enough to ionize the circumstellar nebulae [24].

In order to vary density, we change the filling factor by the power law given by Eq1. Cloudy allows the filling factor which varies with radius to change hydrogen density and to produce hydrogen deficient nebulae [8]. 


$$
f(r)=f\left(r_{o}\right)\left(\frac{r}{r_{o}}\right)^{-\alpha}
$$

Where $r_{0}$ is the inner radius and $\alpha$ the exponent of the power law, in order to have the best agreement between the predicted and observed fluxes, we have to use optimization techniques to search good parameters.

To determine it's calculated a detailed photoionization model with basic assumptions were applied and trying too much with the observed set of line ratios. However, it is very sensitive to physical properties of the nebula such as electron temperature and density, and certain important elemental abundances of planetary nebulae. Therefore, the line ratios can be obtained by enhancing the chemical abundances. Comparing the line fluxes of with the observed fluxes for the nebulae, the possible change of fluxes were observed with the variation of temperatures. The calculations are performed for different sets of emission line intensity ratios corresponding to different cases for comparison shown in Table 2. These fluxes values have been normalized to $\mathrm{H} \beta=100$.

Table 2. The emission line fluxes relative to $H \beta=100$

\begin{tabular}{lllll}
\hline Wavelength [A] & Ions & Observed $^{*}$ & Model 1 $^{*}$ & Model 2 $^{*}$ \\
\hline 6563 & H I & 221.5 & 293.4 & 284.9 \\
6563 & H I & 221.5 & 293.4 & 284.9 \\
4340 & H I & 47.5 & 46.4 & 47 \\
4101 & H I & 21.4 & 25.6 & 21.4 \\
3970 & H I & 13 & 15.7 & 16 \\
5876 & He I & 7 & 4 & 7.19 \\
7065 & He I & 7 & 4 & 1.9 \\
3889 & He I & 13.8 & 2.7 & 4.25 \\
6678 & He I & 3.8 & 1.1 & 2.18 \\
4922 & He I & 3.8 & 0.4 & 0.57 \\
4471 & He I & 8.4 & 1.4 & 1.97 \\
4686 & He II & 4.6 & 0 & 0 \\
4267 & C II & 25.3 & 0.2 & 0.1 \\
5755 & {$[\mathrm{~N} \mathrm{II]}$} & 9.1 & 1.6 & 4.5 \\
6584 & {$[\mathrm{~N} \mathrm{II}]$} & 90.4 & 101.3 & 136.9 \\
6548 & {$[\mathrm{~N} \mathrm{II}]$} & 26.2 & 34.3 & 46.4 \\
6300 & {$[\mathrm{O} \mathrm{I}]$} & 12.9 & 0.9 & 2.12 \\
6363 & {$[\mathrm{O} \mathrm{I}]$} & 4.5 & 0.3 & 0.7 \\
6584 & {$[\mathrm{~N} \mathrm{II]}$} & 90.4 & 101.3 & 136.9 \\
6548 & {$[\mathrm{~N} \mathrm{II}]$} & 26.2 & 34.3 & 46.4 \\
6300 & {$[\mathrm{O} \mathrm{I}]$} & 12.9 & 0.9 & 2.12 \\
6363 & {$[\mathrm{O} \mathrm{I}]$} & 4.5 & 0.3 & 0.7 \\
$3727+29$ & {$[\mathrm{O} \mathrm{II}]$} & 28.2 & 28 & 92.8 \\
5007 & {$[\mathrm{O} \mathrm{III}]$} & 0 & 5.2 & 21.4 \\
6731 & {$[\mathrm{~S} \mathrm{II}]$} & 3.7 & 3.8 & 6.5 \\
\hline
\end{tabular}

*observed data and model 1 were taken from [9].
Table 2 shows the ions in the second column, observed ratios in third column followed by theoretical values of the line ratios as model 1 and model 2 . We note that these line ratios span the optical spectrum.

The result we obtained in this model shown in Table 2 match some of the fluxes predicted by [9] and some of the fluxes do not match observed data. The forbidden line [S II] $\lambda 6731 \mathrm{~A}$ flux greater than both observed and the first model and changes by $41.5 \%$ and $43 \%$ respectively and shown in [9]. These numerical experimental errors suggest that systematic errors may substantially shift the derived abundances or effective temperatures. However, we can see large errors in the forbidden lines of $[\mathrm{N} \mathrm{II}] \lambda 6584 \mathrm{~A}$ relative to model 1 and observed fluxes. These lines predicted by the model presented do not agree with the observed fluxes. This is due to the change of chemical abundances of $\mathrm{N}$, effective temperature and shock waves. These values have an impact on fluxes lines of some ions.

All helium lines excluding He I $\lambda 5876$ are underestimated in the processes. However, in the case of oxygen, the forbidden lines of [O I] are weak in the model but [O II] and [O III] $\lambda 5007$ is relatively stronger than the first model and observed fluxes. This is due to the effective temperature of the star since it is raised by $26.7 \%$ relative to model 1 . This does not mean that they have affected by such change.

A number of models can be constructed by using the values of nebulae radius, density, stellar temperature and chemical abundances. In this work, we basically developed models based on the impact of chemical abundances to get a good result with the observed fluxes. The abundances of $\mathrm{C}$, $\mathrm{N}, \mathrm{O}$, and $\mathrm{S}$ were adjusted to get the best agreement with the observed fluxes of forbidden lines.

Table 3, is model results of fluxes of $\mathrm{C}, \mathrm{N}, \mathrm{O}$, and $\mathrm{S}$ lines shown against abundances of these elements that have been varied by reduction and enhancement by 2 , but the other parameters remain the same as is shown in Table 1. The last two columns are shown in Table 3, the electron temperature and electron density. The result shows that CII flux has peak values only when the carbon abundances enhanced by factors 2 , whereas in the other cases, the abundances of other elements enhanced or reduced by factor 2, CII flux does not show any significant variations.

Table 3. Emission line fluxes showing effect of variation of abundances relative to $H \beta=100$.

\begin{tabular}{|c|c|c|c|c|c|c|c|c|c|c|c|c|c|}
\hline \multirow{3}{*}{ Abundances } & \multicolumn{13}{|c|}{ Flux $[\mathbf{A}]$} \\
\hline & CII & [NII] & & & [OII] & & [OIII] & [SII] & & & & Te [K] & $\mathrm{Ne}\left[\mathrm{cm}^{-3}\right]$ \\
\hline & 4267 & 5755 & 6584 & 6548 & 3727 & 3729 & 5007 & 4078 & 4070 & 6731 & 6716 & & \\
\hline $\mathrm{C}=8.781$ & 0.16 & 4.12 & 130.9 & 44.4 & 66.6 & 18.8 & 19.9 & 4.7 & 14.3 & 6.31 & 2.8 & 7924 & 60420 \\
\hline $\mathrm{C}=8.179$ & 0.04 & 4.83 & 133.9 & 45.4 & 70.2 & 19.8 & 21.0 & 4.59 & 14.1 & 6.22 & 2.75 & 8000 & 60400 \\
\hline $\mathrm{N}=8.61$ & 0.09 & 6.5 & 228.5 & 77.5 & 53.7 & 15.2 & 18.2 & 4.0 & 12.3 & 5.6 & 2.5 & 7777 & 60380 \\
\hline $\mathrm{N}=7.549$ & 0.08 & 2.74 & 71.95 & 22.4 & 78.7 & 22.2 & 22.0 & 5.0 & 15.2 & 6.61 & 2.93 & 8078 & 60390 \\
\hline $\mathrm{O}=8.911$ & 0.083 & 3.63 & 114.7 & 38.9 & 107.2 & 30.3 & 28.8 & 3.86 & 11.8 & 5.47 & 2.43 & 7503 & 60420 \\
\hline$S=7.414$ & 0.082 & 7.86 & 135.6 & 45.8 & 42.9 & 12.1 & 15.7 & 6.9 & 21.2 & 13.6 & 6.1 & 7396 & 60390 \\
\hline$S=6.812$ & 0.083 & 5.54 & 145.3 & 49.2 & 80.1 & 22.63 & 24.2 & 2.5 & 7.65 & 3.32 & 1.47 & 8307 & 60400 \\
\hline
\end{tabular}


However, the other lines fluxes depend on the enhancement and reduction of abundances. As the electron temperature and electron density are shown in the last two columns depend on the abundances.

As the abundances of $\mathrm{C}, \mathrm{N}, \mathrm{O}$, and $\mathrm{S}$ have been varied by enhancement and reduction with 2 , electron temperatures vary by different factors. The change of temperatures is accompanied by the change of chemical abundances. The result shown in Table 3, show that when the chemical abundances of $\mathrm{C}$ changes, its electron temperature changes by $0.95 \%, \mathrm{~N}$ by $3.72 \%$, O by $9.8 \%$ and $\mathrm{S}$ by $11.0 \%$. Among these elements, $\mathrm{S}$ is very sensitive to change electron temperature with the change of abundance.

Fluxes of [SII] $\lambda \lambda \lambda \lambda \lambda 4078,4070,6716,6731 \mathrm{~A}$ highly depend on the enhancement of $\mathrm{S}$ abundances rather than $\mathrm{C}$, $\mathrm{N}$, and $\mathrm{O}$. It is highly sensitive to change line fluxes when the chemical abundances change.
The model of electron temperature is shown in Figure 1. The peak electron temperature is $8.06 \times 103 \mathrm{~K}$. This temperature is less than what was found by [9]. However, the structure of temperature curves is almost similar. It slightly increases and falls sharply to the lowest temperature that is due to heating which is dominated by photoionization of $\mathrm{H}$ and $\mathrm{He}$ and cooling by collisionally excitation of metastable levels of N, O, and $\mathrm{Ne}$ and effect of clouds of dust. For most of the nebulae, at a low temperature of central stars, the electron temperature expected to be in the range of 10000 $17000 \mathrm{~K}$. However, our result shows that the electron temperature is outside this range. There are a number of reasons for this. The first and the most one are heating HI by He II has been ignored because of the low temperature of the star in heating the gas. The electron temperature unrealistically less than the range is given above. The other reason is for such variation is the abundances of elements.

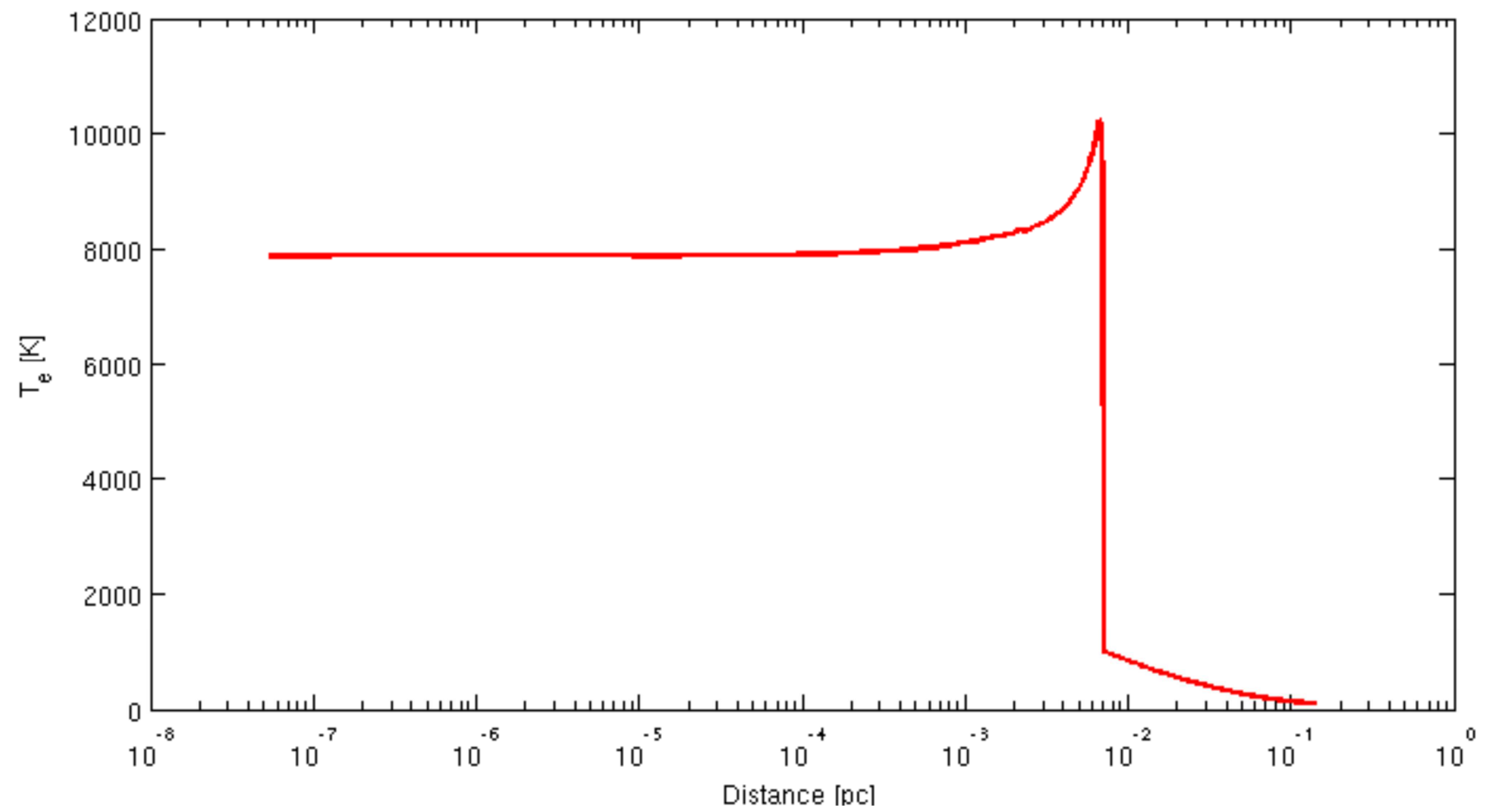

Figure 1. Electron temperature across the nebulae.

Figure 2 shows that the fluctuation of hydrogen density due to the conversion of hydrogen into helium by nuclear interactions. This fluctuation depends on the filling factor and wind speed and it is changed by a factor of $2.7 \%$. Moreover, in this work we assumed the dust grains with PAH and the filling factor which depends on the power index that is given by Eq1, in order to analyze the impact of hydrogen fluctuation with oxygen-rich nebulae on electron density and electron temperature. There is an expansion of the ionized region due to the decreasing density in expanding the shell and increasing the effective electron temperature throughout the region. 


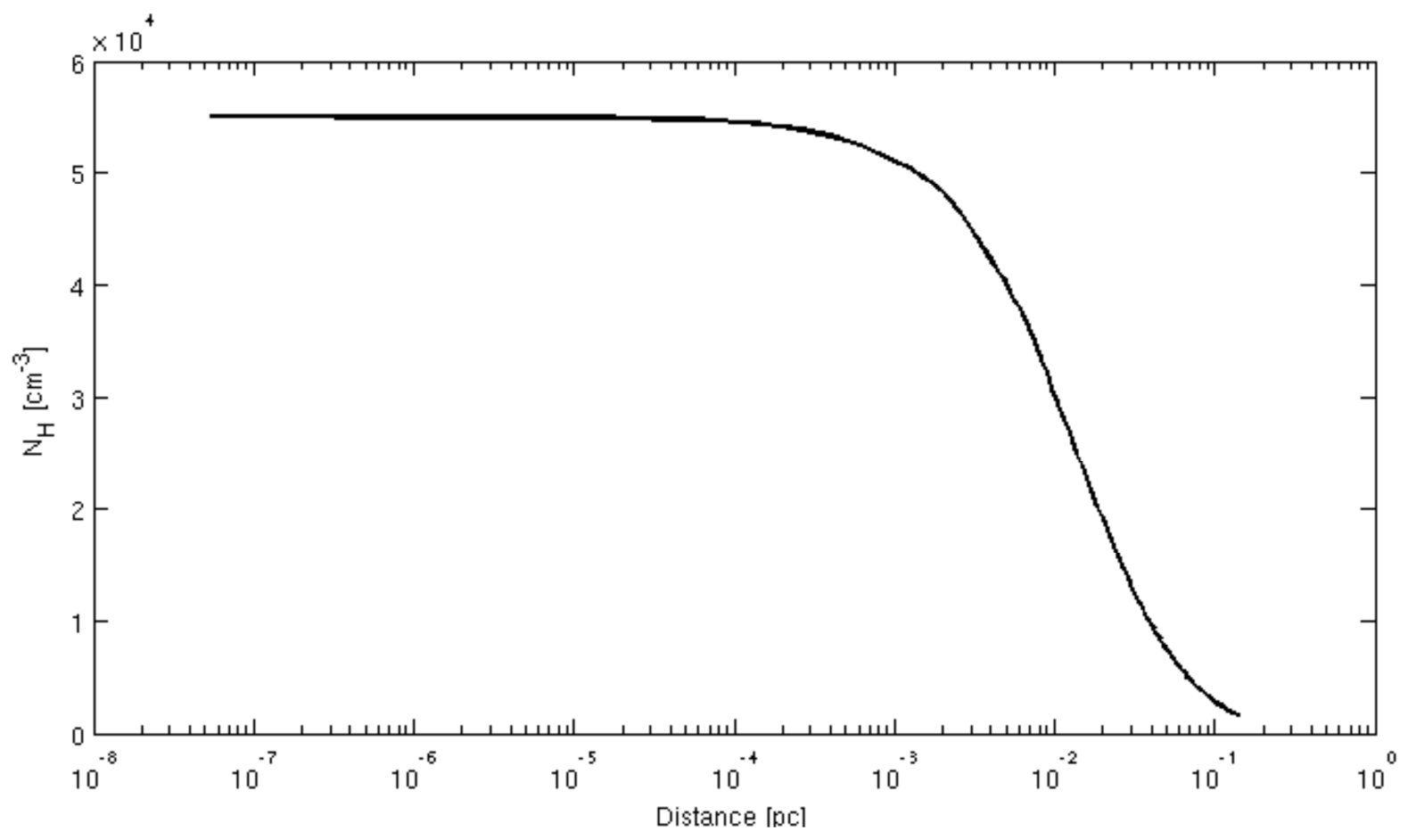

Figure 2. Hydrogen densities across the nebulae.

Figure 3 is electron density. The peak electron density is $5.88 \times 10^{4} \mathrm{~cm}^{-3}$. It has a value that is higher than the electron density calculated by [9]. The electron density totally depends on the abundances of hydrogen and helium. However, this work shows that through the processes hydrogen changed into heavy elements and the contribution of their density to the total impact increases. Due to this, the magnitude of total electron density is slightly increased by $6.54 \%$. The Figure shows that for the large distance from the source decrease slowly and falls sharply as hydrogen density falls with the distance.

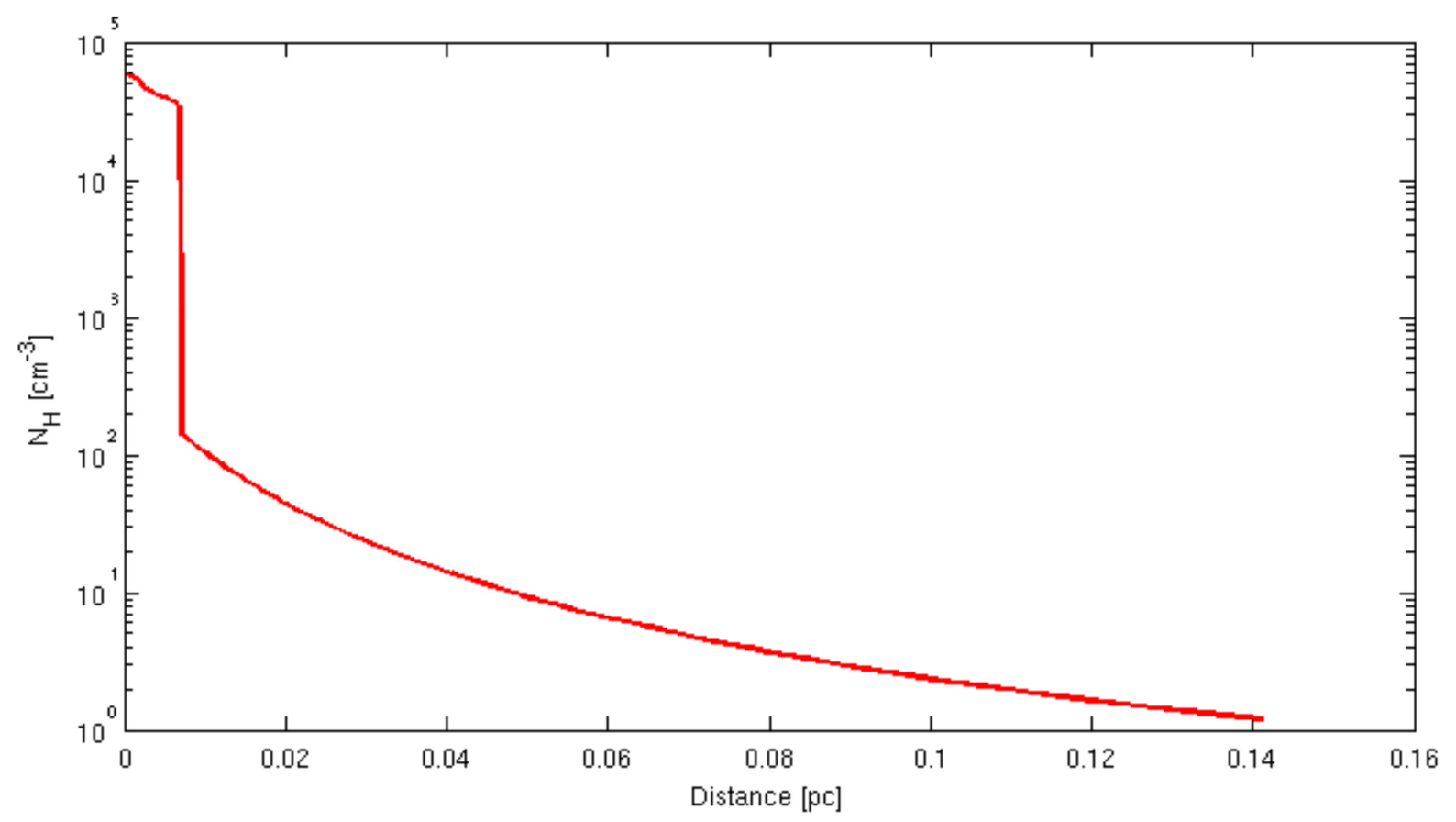

Figure 3. Electron densities across the nebulae.

This result match the result that was described by [34] for lower temperature models the element $\mathrm{H}$ and $\mathrm{He}$ are primarily single ionized, while the heavy elements are predominantly doubly or triply ionized. The ionization structure of heavy elements shown in Figure 4, 5, 6, and 7 agree with [34]. 
The ionization structure of nebulae depends on the energy distribution of its central sources. Therefore, ionization structures of sulfur, nitrogen oxygen, and carbon are given in Figure 4, 5, 6, and 7 respectively. The result shows that in all cases the recombination of ions with ions and with neutral elements is fast. In this model, we have tried to increase the temperature by $1.64 \%$ in order to obtain the best fitting model for fluxes ratios. There is a significant difference between the calculation of oxygen ionization structure by [9] and this model. The ratio of $\mathrm{O}^{2+}$ relative to hydrogen is less than $\mathrm{O}^{+}$initially. The first reason is the abundances of oxygen which is less than [9] and the effective temperature of electrons and wind speed.

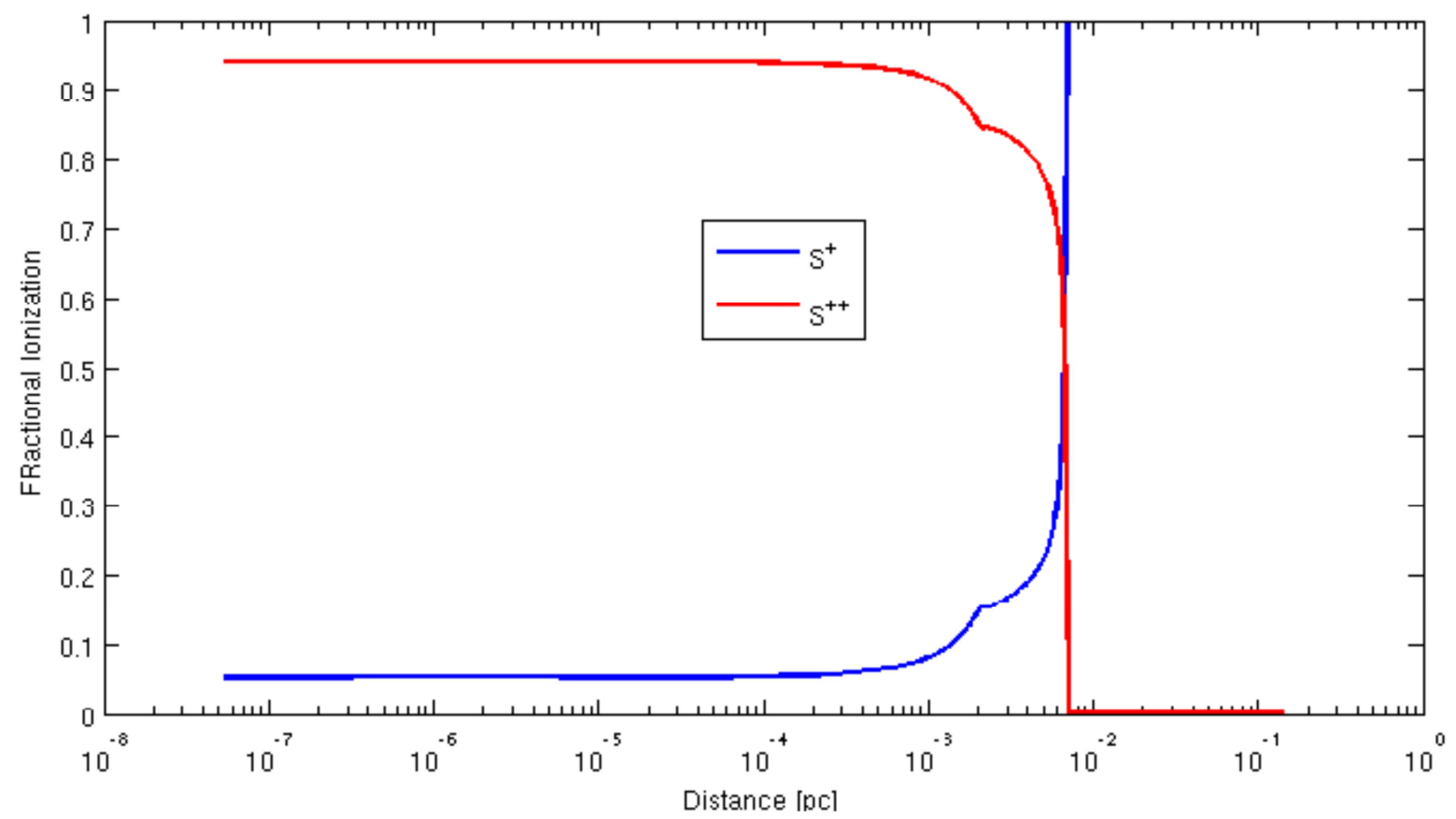

Figure 4. Ionization structures of sulphur atom and ion.

The nebular abundances of elements shown in Table 1 indicates the ratio of $\mathrm{C} / \mathrm{O}$ is 0.74 and it is oxygen rich planetary nebulae. In order to have carbon-rich planetary nebulae, which has ratio $\mathrm{C} / 0=3.6$, which is less than [8]. We increase the abundances of carbon abundances given in Table 1 by $21.0 \%$. The ionization structure of carbon ions that is shown in Figure 7 has similar nature as it is shown in [8].

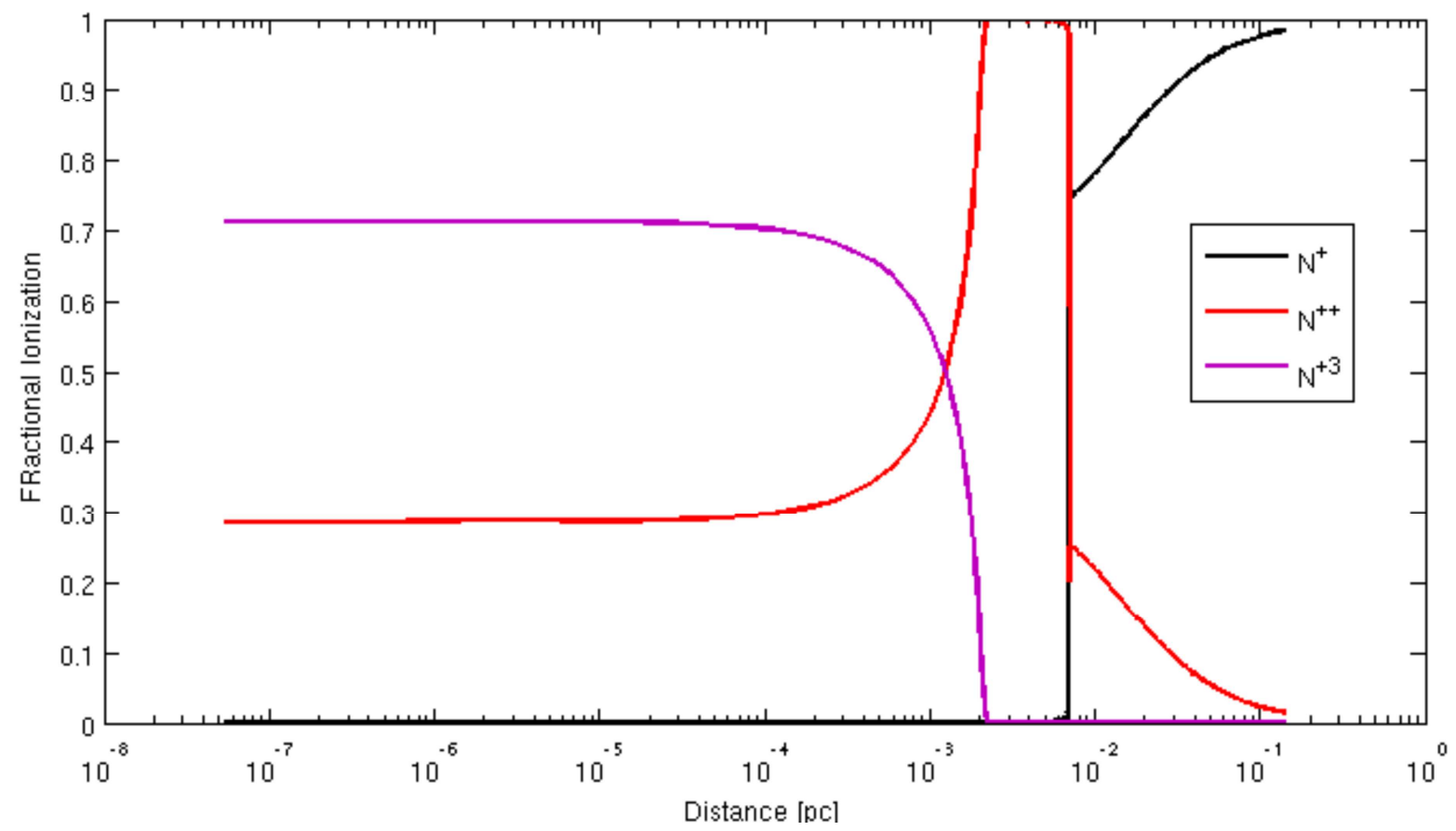

Figure 5. Ionization structures of nitrogen atom and ion. 


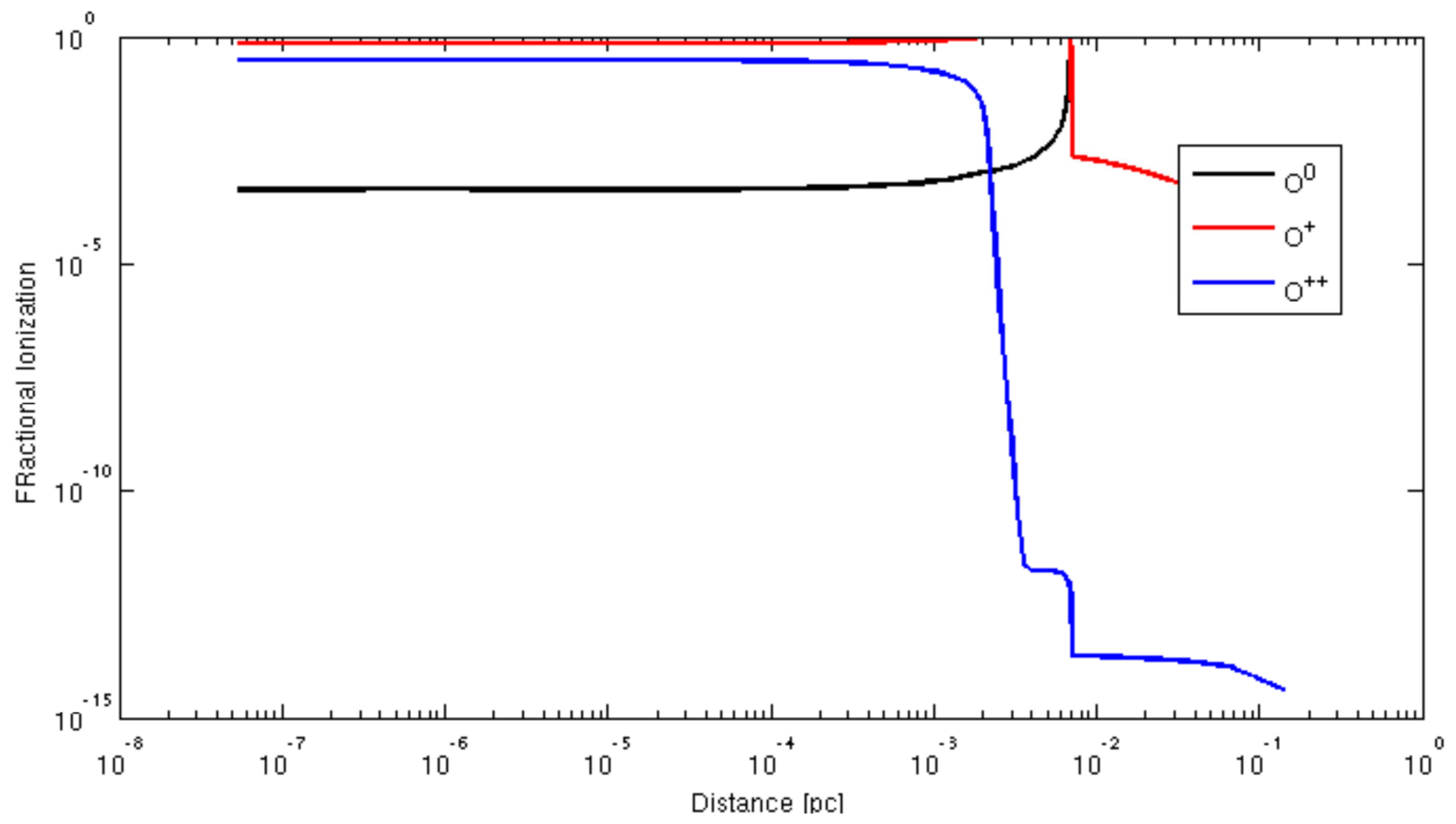

Figure 6. Ionization structures of oxygen atom and ion.

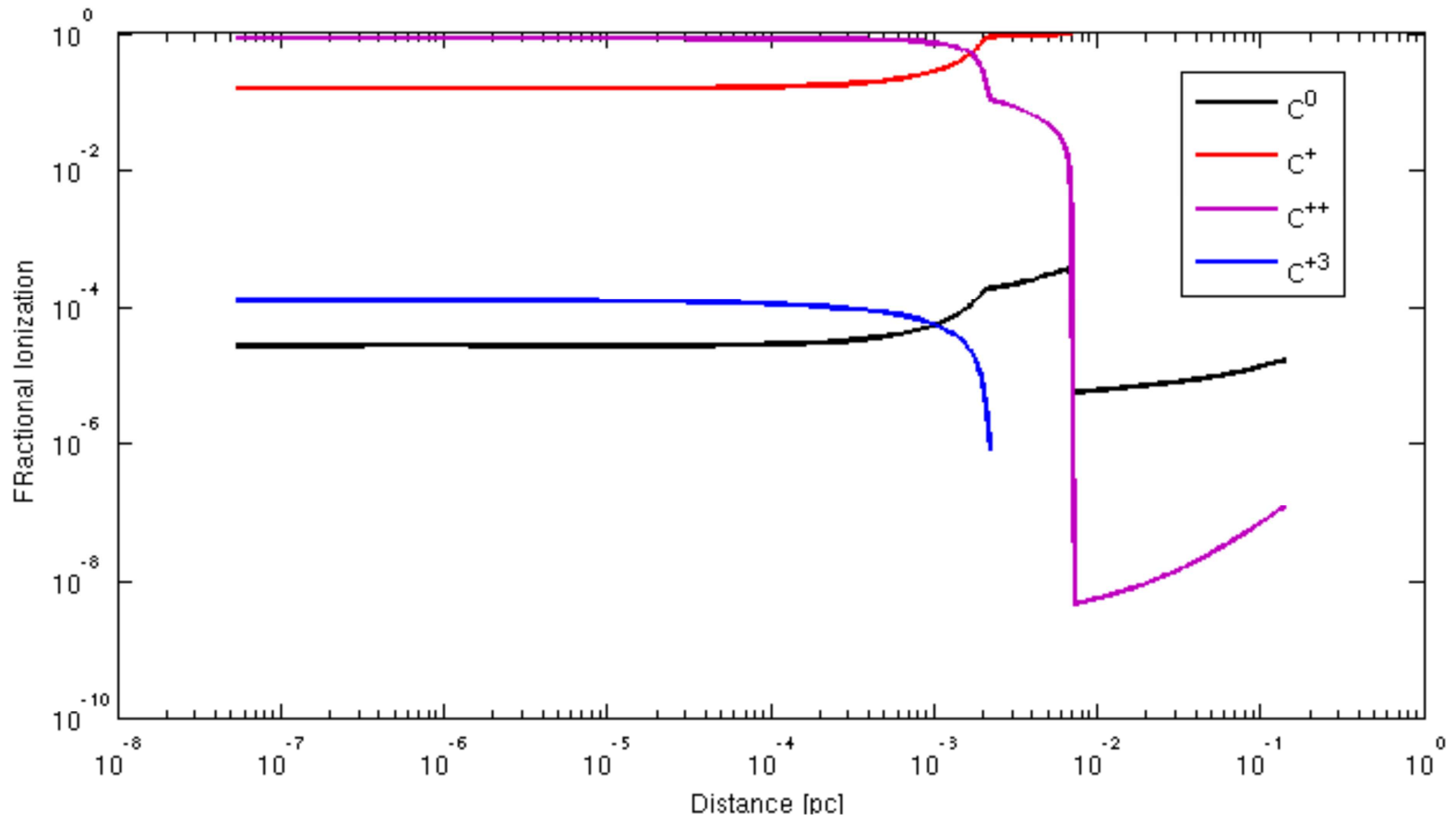

Figure 7. Ionization structures of carbon $\mathrm{C}^{+}, \mathrm{C}^{+2}$, and $\mathrm{C}^{+3}$ ions.

The temperature of grains is shown in Figure 8 . The dust grain Orion peak temperature for single size is $1.86 \times 102 \mathrm{~K}$, which is smaller than the peak temperature of silicon Orion, whereas the peak temperature of PAH is the greatest and has $365 \mathrm{~K}$. The temperature of dust depends on the size of the grain; the large size of the grain is the smaller the temperature and vice versa as it was shown by [29]. This calculation indicates dependent of clouds of dust grain with carbon rich and oxygen rich planetary nebulae. The temperature of dust grains depends on the richness of planetary nebulae. Carbon-rich nebulae temperatures of grain-Orion, silicon-Orion and $\mathrm{PAH}$ change by $0.16 \%$, $0.14 \%$, and $0.05 \%$ respectively. 


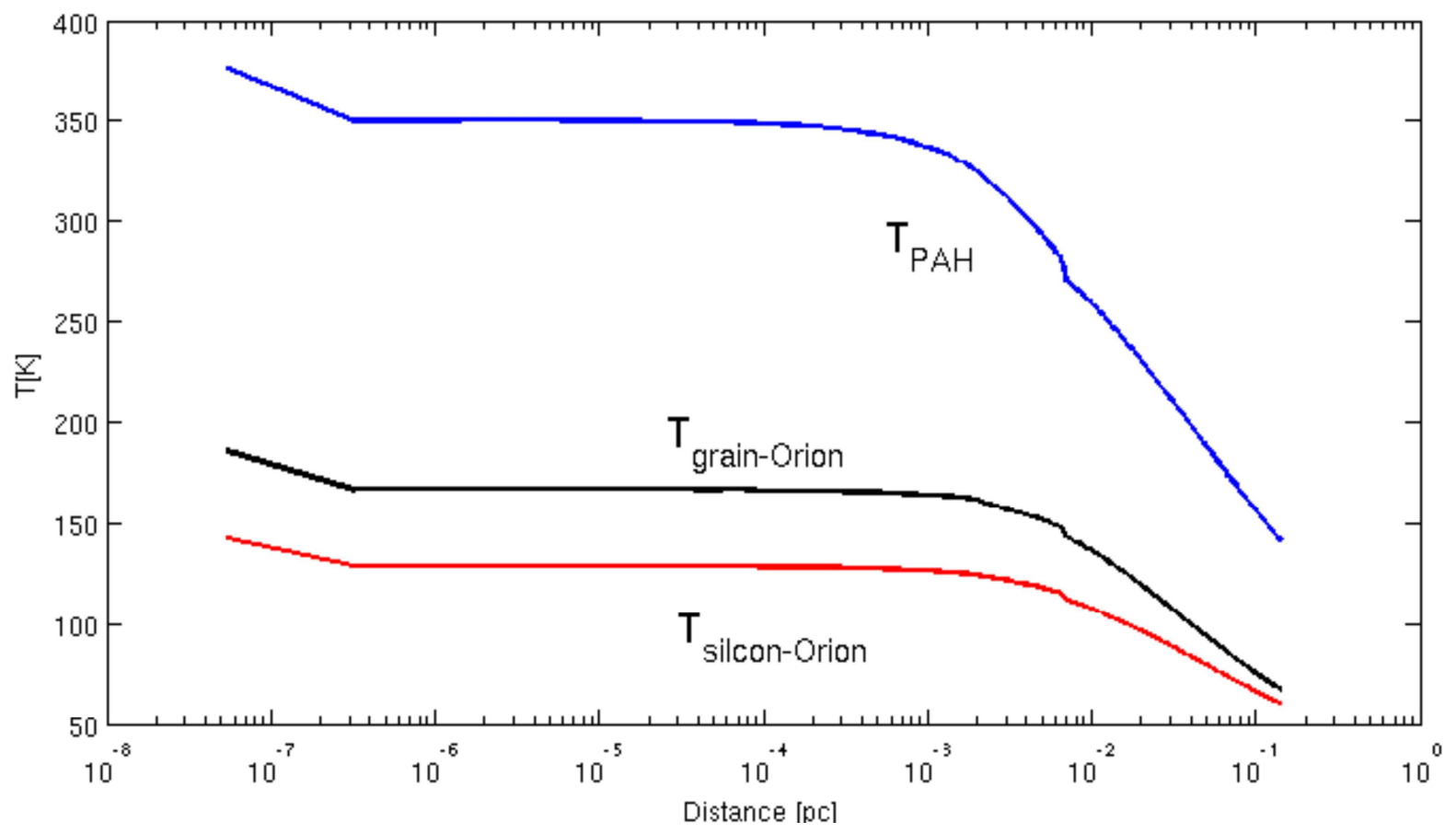

Figure 8. Ionization structures of grains temperatures.

\section{Conclusions}

The main purpose of developing this model is to greatly expand the recent work on planetary nebulae models by creating functionality allowing the existing spectral simulation code Cloudy to produce predicted spectra. Cloudy used to produce the existing emission lines and ionization structure of nebulae with different assumptions and parameters. The photoionization model of IRAS 07027-7934 has been used to study the ionization structure of heavy elements and fluxes ratio using effective temperature, radius, and chemical composition. We discuss the possibility of determining the fluxes ratio of elements relative to $\mathrm{H} \beta$ and the other possibility is to evaluate the ionization structure of heavy elements at the low temperature of stars. The analysis presented in this work is feasible to demonstrate fluxes ratio with different assumptions and values of chemical composition, temperature, filling factor which decays by power law given by Eq 1 and stellar winds speed. We have used these assumptions for these particular planetary nebulae IRAS 07027-7934.

It has shown that the fluxes ratio of HI $\lambda \lambda 4340 ; 4101 \mathrm{~A}$, and HeI $\lambda 5876 \mathrm{~A}$ match observed data shown in Table 2. The test data do not exactly match the observed, but it is better than the calculated values of the previous work.

Finally, in this work it has calculated electron temperature, density and fluxes of different ions by enhancing and reducing carbon abundance by the factor of 2 , CII flux does not show any significant variations. However, all the other lines fluxes depend on the enhancement and reduction of abundances. As the electron temperature and electron density are shown in the last two columns depend on the abundances. Among $\mathrm{C}, \mathrm{N}, \mathrm{O}, \mathrm{S}$ abundances, $\mathrm{S}$ is very sensitive to change electron temperature with the change of abundance. Fluxes of [SII] $\lambda \lambda 4078 \mathrm{~A}, 4070 \mathrm{~A}, 6716 \mathrm{~A}, 6731 \mathrm{~A}$ highly depends on the enhancement of S abundances rather than $\mathrm{C}, \mathrm{N}$, and $\mathrm{O}$. S is highly sensitive to the chemical abundances.

\section{References}

[1] Garci'a-Hern'andez D. A., Manchado A., Garci'a-Lario P., Beni'tez Ganete A., Acosta-Pulido J. A., \& Pi'erezGarci'a, Revealing the mid-infrared emission structure of IRAS 16594-4656 and IRAS 07027-7934, 2005, arxiv:astro$\mathrm{ph} / 0512064 \mathrm{v} 1$.

[2] García-Hernández D. A., P. García-Lario P., B. Plez B., A. Manchado A., and D'Antona F., Lub J. and H. Habing H., 2005, Why are massive O-rich AGB stars in our Galaxy not Sstars?, arxiv:astro-ph/0509051v1.

[3] Balick B., \& Frank A., Shapes and Shaping of Planetary Nebulae, 2002, ARA\&A, 40, 439.

[4] Van Winckel H., Post-AGB Stars, 2003, ARA\&A, 41, 391.

[5] Morris M., Mechanisms for mass loss from cool stars, 1987, PASP, 99, 115.

[6] Soker N., \& Kastner J. H, Magnetic Flares on Asymptotic Giant Branch Stars, 2003, ApJ, 592, 498.

[7] Garci'a-Segura G., Langer N., \& Rozyczka M., Shaping Bipolar and Elliptical Planetary Nebulae: Effects of Stellar Rotation, Photoionization Heating, and Magnetic Fields, 1999, ApJ, 517, 767.

[8] Pascoli G., On Planetary Nebulae formation, 1992, PASP, 104, 350 .

[9] Surendiranath R., Deconstructing IRAS 07027-7934, 2002, A\&A 390, 667. 
[10] Menzies J. W., \& Wolstencroft R. D., IRAS: 07027-7934 - a Probable New WC11 Sta, 1990, ApJS, 122, 221.

[11] Gesicki K., Zijlstra A. A., Acker A, Górny S. K., Gozdziewski K., and Walsh J. R., 2006, Planetary nebulae with emissionline central stars, A\&A 451, 925-935.

[12] Baldwin J. A., Ferland G. J., Martin P. G., Corbin M., Cota, S., Peterson B., \& Slettebak A., Physical conditions in the Orion Nebula and an assessment of its helium abundance, 1991, ApJ, 374, 580.

[13] Bains I, Richards A. M. S., T. M. Gledhill T. M., and J. A. Yates J. A., MERLIN polarimetry of the $\mathrm{OH}$ masers in IRAS 20406+2953, 2004, MNRAS, 354, 529-542.

[14] Engels D. and Bunzel F., 2015, A database of circumstellar OH masers, arxiv: 1508. 062200v2, [astro-ph. SR].

[15] Zijlstra A. A., Te Lintel Hekkert P., Pottasch S. R., Caswell J. L., Ratag M., \& Habing H. J., OH maser emission from young planetary nebulae, 1989, A\&A, 217, 157.

[16] Corradi R. L. M., Perinotto M., Schwarz H. E., \& Claeskens JF., The chemical structure of bipolar planetary nebulae. I. IC 4406, 1997, A\&A, 322, 975.

[17] Kwitter K. B., M'endez R. H., Pẽna M., Stanghellini L., Corradi R. L. M., De Marco O., Fang X., Henry R. B. C, Karakas A. I., Liu X. W., L'opez J. A., Manchado A., and Parker Q. A., 2014, The present and future of planetary nebula research. A white paper by the iau planetary nebula working group, Revista Mexicana de Astronom'1a y Astrof'1sica, 50, 203-223.

[18] Wesson R., Barlow M. J., Liu X-W., Storet P. J., Ercolano B., \& De Marco O., The hydrogen-deficient knot of the 'bornagain' planetary nebula Abell 58 (V605 Aql), 2008, MNRAS 383, 1639-1648.

[19] Habing H. J., van deer Ven W., \& Geballe T., 1987, in the late stages of stellar evolution, ed. Volk S. R., Pottasch S. R., (Dordrecht: Raidel), p, 91.

[20] Volk K. M., \& Kwok S., Spectral evolution of asymptotic giant branch stars, 1988, ApJ, 331, 435.

[21] Ferland G. J., et al. 2013, Hazy: Introduction to cloudy c13.03.

[22] Pequignot, D; Amara, M; Liu, XW; Barlow, MJ; Storey, PJ; Morisset, C; Torres-Peimbert, S; (2002) Photoionization models for planetary nebulae with inhomogeneous chemical composition. Ionized Gaseous Nebulae, 12 142-143.

[23] Henry R. B. C., \& Worthey G., The Distribution of Heavy Elements in Spiral and Elliptical Galaxies, 1999, PASP, 111, 919 .

[24] Phillips, J. P., The mean properties of planetary nebulae as a function of Peimbert class, 2005 MNRAS 361, 283-299.

[25] Pilleri, P., Herberth, D., Giesen, T. F., et al. Search for corannulene $\left(\mathrm{C}_{20} \mathrm{H}_{10}\right)$ in the Red Rectangle 2009, MNRAS, $397,105$.

[26] Weingartner, J. C. \& Draine, B. T. Dust Grain-Size Distributions and Extinction in the Milky Way, Large Magellanic Cloud, and Small Magellanic Cloud, 2001a, ApJ, 548, 296.

[27] Harrington J. P., Monk D. J., \& Clegg R. E. S., Thermal infrared emission by dust in the planetary nebula NGC 3918 A model analysis of IRAS observations, 1988, MNRAS, 231, 577.

[28] Hoare M. G., \& Clegg R. E. S., A silicate dust model for the halo planetary DDDM-1, 1988, MNRAS, 235, 1049.

[29] Asplund, M., Grevesse N., Sauval A. J., \& Scott, P. 2009, The Chemical Composition of the Sun, ARA\&A, 47, 481.

[30] Osterbrock D., Tran H., \& Veilleux S., Faint emission lines in the spectrum of the Orion Nebula and the abundances of some of the rarer elements, 1992, ApJ, 389, 305.

[31] Henry R. B. C, Kwitter K. B, Anne E. Jaskot A. E., Balick B., Morrison M. A., and Milingo J. B., 2010, Abundances of galactic anticenter planetary nebulae and the oxygen abundance gradient in the galactic disk, The Astrophysical Journal, 724:748-761.

[32] Rubin R. H., Colgan S., Dufour R., \& Lord, S., The $\mathrm{He}^{+} / \mathrm{H}^{+}$ Abundance in the Orion Nebula from Infrared Space Observatory Measurements, 1998, ApJ, 501, L209.

[33] Shields J. C., \& Kennicutt R. C., Consequences of Dust in Metal-rich H II Regions, 1995, ApJ, 454, 809.

[34] Williams R. E., in IAU Symp. 34, Planetary Nebulae, ed. Osterbrock, D. E. \& O'Dell, C. R. (New York, SpringerVerlag), 190, 1968, IAUS, 34, 190. 